\title{
Students' Use of Computational Thinking in Linear Algebra
}

\author{
Spencer Bagley ${ }^{1}$ • Jeffrey M. Rabin ${ }^{2}$
}

Published online: 25 November 2015

(C) Springer International Publishing Switzerland 2015

\begin{abstract}
In this work, we examine students' ways of thinking when presented with a novel linear algebra problem. Our intent was to explore how students employ and coordinate three modes of thinking, which we call computational, abstract, and geometric, following similar frameworks proposed by Hillel (2000) and Sierpinska (2000). However, the undergraduate honors linear algebra students in our study used the computational mode of thinking in a surprising variety of productive and reflective ways. This paper examines the solution strategies that the students employed to solve the problem, emphasizing their use of the computational mode of thinking.
\end{abstract}

Keywords Justification · Linear algebra · Problem solving · Procedural understanding · Conceptual understanding

\section{Introduction}

A first course in linear algebra plays a pivotal role in the mathematical education of college students in STEM disciplines. It usually follows a calculus sequence that may be predominantly computational in focus, and serves as a first encounter with many elements of more advanced mathematics. These include the emphasis on precise definitions and formal proofs, the creation of an abstract axiomatic structure, the study of objects that are not easy to visualize, multiple representations of those objects, and computational methods that require non-routine choices and interpretations of those

Spencer Bagley

spencer.bagley@unco.edu

Jeffrey M. Rabin

jrabin@ucsd.edu

1 School of Mathematical Sciences, University of Northern Colorado, Campus Box 122, 501 20th Street, Greeley, CO 80639, USA

2 Department of Mathematics, UC San Diego, 9500 Gilman Drive \# 0112, La Jolla, CA 92093-0112, USA 
representations. The kinds of flexible thinking required are foreign to many students, for whom "the fog rolls in" and the subject remains opaque (Carlson 1993).

Much of the literature on the learning of linear algebra documents students' inabilities to solve basic problems, to move flexibly between the representations, to use abstract theorems in concrete situations, and even to speak or write the basic language of linear algebra coherently. Despite much insight into the causes of their difficulties, and creative pedagogical suggestions, the overall impression remains pessimistic.

Based on our own teaching experience, and consistent with previous work (Hillel 2000; Sierpinska 2000), we hypothesized that students must learn three major ways of thinking, and how to coordinate them with each other, in order to succeed in linear algebra. We term these abstract, geometric, and computational thinking. The study reported here was designed to explore students' use of these ways of thinking and their ability to coordinate them while solving a novel linear algebra problem. The initial research questions for the study were: What strategies do students use to solve a novel linear algebra problem (described below)? What are the uses, affordances, and constraints of each mode of thinking? In what ways do students coordinate these modes of thinking? As our analysis progressed, we noted a preponderance of computational thinking, used by students in unexpectedly effective ways, and we were thus led to ask in addition: In what productive ways did students use computational thinking? The present paper concentrates on this question.

The students in our study were first year undergraduates enrolled in an Honors linear algebra course at a large university in the southwestern United States. As such, they do not form a representative sample of the general population of linear algebra students, and we would not expect our results to generalize immediately to this larger population. However, our students successfully used computational thinking to solve the problem in creative ways and to justify their solutions. Our study shows what successful student thinking in linear algebra can look like and provides a positive counterpoint to the literature on student deficiencies in linear algebra.

\section{Theoretical Background and Literature Review}

Drawing upon our own teaching experience, we hypothesize that mastery of linear algebra requires students to develop, and move flexibly between, three essential ways of thinking that we term abstract, geometric, and computational thinking. Our tripartite taxonomy of modes of thinking is closely related to similar categories described by Hillel (2000) and Sierpinska (2000). We now review their work and explain how our own viewpoint builds upon it.

Hillel speaks of three modes of description of vectors and operators: the abstract, algebraic, and geometric modes. He defines the abstract mode as using the language and concepts of the general formalized theory, including vector spaces, subspaces, linear span, dimension, operators, and kernels. His algebraic mode includes the language and concepts of the more specific theory of $\mathbf{R}^{\mathrm{n}}$, including $\mathrm{n}$-tuples, matrices, rank, solutions of systems of linear equations, and row space. Even more specifically, his geometric mode includes the language and concepts of 2and 3-space, including directed line segments, points, lines, planes, and geometric transformations. 
Hillel (2000) traces many student difficulties to instructors' propensity for "constantly shifting modes of description and notation" (p. 199) without alerting students to this. He points out that choosing a basis, or changing basis, may require students to shift modes of description, and that over-reliance on a single mode can cause problems for students, for example when they inappropriately apply geometric intuition in dimensions higher than three. His three modes roughly correspond to ours, but refer to languages that can describe the basic objects of study, whereas ours refer directly to the ways that students think; Hillel acknowledges that "it is possible, for example, for students to be working in $\mathbf{R}^{\mathrm{n}}$ (the algebraic mode of description) and to be using several modes of reasoning" (p. 195).

Sierpinska (2000), like us, discusses three modes of thinking or reasoning. We will shorten her names to (analytic-)structural, (synthetic-)geometric, and (analytic-)arithmetic. While she does not give explicit definitions of these categories, she provides a wealth of examples. Her categories roughly correspond to Hillel's abstract, geometric, and algebraic modes, respectively. She says these modes are "equally useful, each in its own context, and especially when they are in interaction" (p. 233) and traces student difficulties to "their inability to move flexibly between the three modes" (p. 209). Her structural mode corresponds to our abstract one, and her geometric mode to ours. However, we intend our computational mode to be more inclusive than her arithmetic mode. As we elaborate below, our computational mode includes reasoning about computations, which Sierpinska views as overlapping with the other modes of thinking. Such computational reasoning, as we call it, will be a central theme of this paper.

\section{Our Tripartite Taxonomy}

We use a similar threefold classification of students' modes of thinking, comprising abstract, geometric, and computational thinking; we present examples of each in the Results section. Abstract thinking treats vectors as abstract objects manipulated according to formal rules, without reference to components or arrows. It makes use of definitions and theorems stated in coordinate-free language and applicable to $\mathbf{R}^{n}$ for any $n$. (Our course did not cover abstract vector spaces.) It makes assertions of existence or uniqueness on the basis of general principles rather than resulting from explicit computations. The notion of orthogonality may be part of abstract thinking if its meaning comes from an abstract inner product rather than the specifically geometric notion of right angles.

Geometric thinking involves visualization in Euclidean two- or threedimensional space, with vectors represented as arrows, and may draw on concrete facts from high school geometry such as the Pythagorean Theorem. Span and linear independence can be geometric concepts if based on geometric properties such as collinearity and coplanarity in $\mathbf{R}^{2}$ and $\mathbf{R}^{3}$. "Orthogonal" as a synonym for "perpendicular" is a geometric concept. Systems of linear equations can be viewed geometrically in terms of the incidence relations among the lines or planes obtained by graphing each equation. A desirable outcome of a linear algebra course is for students to learn to extend their geometric thinking to apply in $\mathbf{R}^{n}$ for $n>3$; we do not consider "geometric thinking" in such contexts to be an oxymoron. 
Since the present study emphasizes students' use of computational thinking, we wish to focus on our definition of computational thinking and its relation to Sierpinska's (2000) arithmetic mode of thinking. Computational thinking represents vectors in $\mathbf{R}^{n}$ explicitly as $\mathrm{n}$-tuples of real numbers, and draws conclusions from algorithms such as row reduction of matrices for solving systems or computing determinants. Assertions of existence or uniqueness come from explicit computations producing the objects in question. Systems of linear equations are thought of in terms of their coefficient or augmented matrices. Most importantly, for us computational thinking is not simply the procedural knowledge of how to execute an algorithm without errors. It includes choosing the appropriate computation to answer a particular question, understanding what the result of the computation means in that context, and reasoning about the steps or the outcome of the computation.

In essence, our computational mode of thinking is an expansion of Sierpinska's (2000) arithmetic mode to include reasoning about computations. Although Sierpinska includes some reasoning and proof within her arithmetic mode of thinking, its precise limits are not clear to us, and seem too restrictive. On the one hand, she says that "Much of the analytic-arithmetic reasoning goes along the line: Show that two processes lead to the same result" (p. 234). On the other, she gives an example she considers to be intermediate between arithmetic and structural thinking, in which "the student was not performing calculations, he was reflecting on the properties of the possible effects of a calculation" (p. 240). We definitely consider this within the scope of computational thinking. Regarding a second example of student reasoning, about the algorithm for inverting a matrix, she says that it is partly arithmetic but has "one foot in the structural mode because it reflects on the technique and makes use of its reversible character" ( $p$. 241). We would again consider this within the scope of computational thinking.

Problems in linear algebra may require an insight or reasoning process that is most accessible via one specific way of thinking. However, coordination between multiple ways of thinking is often required. By this we mean the ability to flexibly move from one way of thinking to another, or more specifically to "import" information acquired via one mode into another mode for further reasoning. Translation to the geometric mode often provides intuitive confirmation or understanding of abstract or computational results; a geometric picture may provide the key idea for an abstract proof or identify a key quantity to be computed. For instance, a geometric viewpoint on least squares and orthogonal projection is an invaluably illuminating complement to an abstract or computational one. The abstract mode can provide necessary or sufficient conditions for drawing some conclusion, which conditions can then be verified computationally. Our study was initially designed to explore students' ability to coordinate multiple modes of thinking in solving a novel problem, and the affordances provided by such coordination, which is a feature of "expert" thinking in linear algebra. In delineating the "boundaries" between the modes of thinking, and studying their interaction at these boundaries, we consider it important that each mode be defined broadly enough to include reasoning processes carried out within its own "language". In particular, this is the reason for our expansion of Sierpinska's (2000) arithmetic mode of thinking into our computational category.

We view the classification of student thinking in linear algebra as computational, geometric, or abstract as being largely orthogonal to the broader classification of students' knowledge as procedural or conceptual. Students thinking in any of the 
modes may draw upon knowledge anywhere on the continuum from procedural to conceptual. In particular, although computational thinking would include superficial procedural knowledge, in which students apply rote algorithms without reflection (Hiebert and Lefevre 1986), we do not view computational thinking as limited to superficial procedural knowledge. At its best, computational thinking can be an example of what Baroody et al. (2007), responding to Star (2005), call deep procedural knowledge. For Baroody et al. (2007), "(relatively) deep procedural knowledge cannot exist without (relatively) deep conceptual knowledge" (p. 123). The "procedural flexibility and critical judgment" that for us can be exhibited by students using computational thinking "require the integration of procedural knowledge with conceptual knowledge" (Baroody et al., p. 121). We will present examples of our students successfully and flexibly applying computational thinking to solve the problem we posed in a variety of creative and reflective ways.

\section{Literature Review}

The early literature on linear algebra tends to emphasize identification and diagnosis of student errors and deficiencies. Hillel (2000) and Sierpinska (2000) highlight students' inability to utilize and flexibly coordinate the three modes of thinking. Dorier and Sierpinska (2001) also point out students' unfamiliarity with axiomatic frameworks and the need to think in terms of formal definitions and general properties. Maracci (2008) studied student work on a challenging problem about the intersection of subspaces of a vector space of dimension at least 5 . He suggested that their difficulties reflect an insufficiently general "paradigmatic model" of subspace limited to the span of a selected subset of given basis vectors, like the coordinate subspaces $x_{i}=0$ of $\mathbf{R}^{\mathrm{n}}$. He also pointed out the need to view a linear combination both as a process and as an object. Stewart and Thomas (2010) frame their work in terms of APOS theory and Tall's three worlds of mathematics (embodied, symbolic, and formal) which they compare to Hillel's three modes of description. They point out that students are often not given time and opportunities to develop links between the three worlds, and that their procedural knowledge is not deep in the above sense: "students who thought that they should row reduce a matrix often did not know why, or what to do with the result" (p. 186).

More recent work presents a more optimistic picture of student understanding in linear algebra. Several studies have explored sociocultural aspects of student learning, and proposed new instructional approaches to enrich students' concept images in linear algebra. Wawro (2014) documented the evolution of student argumentation, at the level of the classroom community, concerning the Invertible Matrix Theorem over the course of a semester. This provides insight as to how students use and modify their understanding of key concepts like span and independence as they make connections between the many criteria for a matrix to be invertible. Wawro et al. (2012) described the implementation of an instructional sequence in the spirit of Realistic Mathematics Education to build rich concept images for span and independence, in which vectors are identified with modes of travel through space. They showed that students continue to draw on these concrete images later as they work on more abstract tasks. Possani et al. (2010) similarly used a realistic traffic modeling task to generate concept images for systems of linear equations and their solution methods. 
Student problem-solving has also been examined in the framework of Sierpinska's three modes of thinking. Dogan-Dunlap (2010) coded students' different approaches to determining whether given sets of vectors were linearly independent and categorized these within Sierpinska's three modes of thinking. Students' use of geometric thinking was of most interest inasmuch as they were provided with software generating visual representations of the vectors in question. Celik (2015) similarly coded approaches to a more abstract task asking whether the (in)dependence of a set of three unspecified vectors implies the (in)dependence of the new set obtained by deleting one vector, or by adding another. This latter task has some relation to our task (see below) of extending a given set of vectors to a basis. Both of these authors found that arithmetic (computational) thinking predominated, which is consistent with our finding here.

\section{Materials and Methods}

The students in our study were enrolled in an Honors Linear Algebra course in their first year at a large university in the southwestern United States. The course forms the first quarter of a three-quarter Honors Calculus sequence in which linear algebra provides the conceptual basis for treating multivariable calculus in any number of dimensions. Completion of Advanced Placement (AP) calculus in high school, and obtaining the highest possible score of 5 on the AP calculus $\mathrm{BC}$ examination, are prerequisites for the course. The instructor was not an author of this paper, although we did observe his class on a few occasions and asked him some questions about the course content. One of us has taught the course in previous years, including the year of the pilot study mentioned below. Both the textbook (Hubbard and Hubbard 2009) and the instructor of this iteration of the course take a fairly computational approach to linear algebra, building much of the subject around the key idea of finding solutions to linear systems.

Eight students (of 34 enrolled) responded to our request for volunteers to participate in a clinical interview (Ginsburg 1997); all of these were accepted. The eight students' course grades ranged from A though $\mathrm{C}$. Although all volunteers were male, this was not unrepresentative of the class, which included only a few female students. Students were interviewed individually; the interviews lasted about one hour and took place at the end of the course, when essentially all course material had been covered.

The interview revolved around the following task, termed the "Michelle Problem:"

Michelle would like to create a basis for $\mathbf{R}^{4}$. She has already listed two vectors $\mathbf{v}$ and $\mathbf{w}$ that she would like to include in her basis, and wants to add more vectors to her list until she obtains a basis. What instructions would you give her on how to accomplish this?

We had included this problem as one among many interview questions in an earlier pilot project conducted near the end of a previous year's iteration of the same honors linear algebra course (Wawro et al. 2011). Based on the responses at that time, we determined that the task has the potential to elicit all three modes of thinking: computational, geometric, and abstract. Although some textbooks do prove that a linearly independent set of vectors can always be extended to a basis, this was not 
covered in the course the students took, and thus the problem was novel to them. The framing in $\mathbf{R}^{4}$ rather than $\mathbf{R}^{3}$ is intended to discourage an immediate appeal to visual geometric intuition, although geometric thinking is still applicable. Not providing numerical components for the "given" vectors may facilitate an abstract approach, and by asking for "instructions" for Michelle we hope that students will reflect on their methods and perhaps present them in algorithmic form. The semistructured interviews were conducted by one of the authors, joined by another colleague on some occasions.

Most of the eight students did not make much progress on the problem in the very general way in which it was initially presented, with generic vectors $\mathbf{v}$ and $\mathbf{w}$. When they appeared "stuck" we asked them to test, or continue to develop, their ideas using the specific vectors $\mathbf{v}=\left[\begin{array}{llll}1 & 2 & 3 & 4\end{array}\right]$ and $\mathbf{w}=\left[\begin{array}{lll}0 & -1 & 4\end{array}\right]$. We asked a number of follow-up questions to probe students' intuition and solution procedures, whether students could formulate their solution procedure in an algorithmic way, and whether they could justify their procedure. More details of the interview protocol are provided in Appendix 1.

The interviews were videotaped and transcribed, and students' written work produced during the interviews was retained. These recordings, transcripts, and written documents formed the corpus of data analyzed in this study. Using grounded theory (Strauss and Corbin 1994), we coded students' utterances as instances of abstract, computational, or geometric thinking, referring to written work for confirmatory evidence, and documented the ways in which students used each mode of thinking. To code students' utterances, we attended to vocabulary indicative of specific modes; for example, we usually coded utterances including "plane" and "perpendicular" as instances of geometric thinking, whereas we usually coded utterances including "pivot" and "row reduction" as instances of computational thinking. Some terms, especially "orthogonal", are meaningful in any mode, and we used context to resolve such ambiguity. As an example of the use of context, two students in our pilot study used the term "perpendicular" in the context of taking the cross product of two vectors or of the Gram-Schmidt process; based on the context, we could not conclude that they were thinking geometrically, since it was just as likely that they were thinking about an algorithm. References to the components of vectors, or the entries of matrices, were usually taken to indicate the computational mode. We coded statements of theorems or properties in coordinate-free language as instances of abstract thinking. We also attended to notations, procedures, images, or metaphors students used. In particular, our students often explicitly carried out the steps of an algorithm such as row reduction, or explicitly reasoned about those steps; this was one of our primary indications that they were using the computational mode of thinking. Similarly, reasoning based on visual images or Euclidean geometry provided evidence of geometric thinking.

\section{Results}

The students in our interviews were largely successful in solving this task; all but one of the eight students developed a solution procedure. The most frequently used solution method was some variant on the guess-and-check method; however, students usually developed strategies for producing guesses that they thought were more likely to succeed. A summary of solution methods and the number of students who used each 
is presented in Appendix 2. Throughout this section, we will capitalize the name of each mode of thinking to more clearly mark them.

Most of our results document students using Computational thinking in ways we found surprisingly effective. As a contrast to those results, and to exemplify our coding, we begin by documenting some productive ways students used Abstract and Geometric thinking. Most of the students began with the Abstract definition of basis before shifting into another mode of thinking. For instance, Carl's ${ }^{1}$ initial response was, "She would just start by finding one vector that was linearly independent, that could not be made as a combination of $\mathbf{v}$ and $\mathbf{w}$, and once she found that vector, she would have to find another one that was not a linear combination of the other three." This was the only consistent use of Abstract thinking across the students in our study. Once they were given specific numerical vectors, students tended to quickly shift into Computational thinking.

We had anticipated that the notion of orthogonality would be most likely to lead students to think Geometrically, for example recommending that Michelle choose vectors orthogonal to those she already has. To our surprise, it was an intuitive idea of probability, or "measure zero", that led one student in this direction. For instance, when Bob was asked how many bases meet Michelle's requirements, he said:

$B o b$ : She would just pick a third vector that's not in the same plane as the other ones. Which, if you're talking about comparing infinities, we're talking about a little infinity for just that plane, versus the huge infinity that's the rest of $\mathbf{R}^{3}$. So in $\mathbf{R}^{4}$ would be the same; there's a little three-dimensional space, and that the rest of $\mathbf{R}^{4}$ is huge, that you can choose from.

Here, Bob attacked the problem Geometrically, by visualizing the relative sizes of $\mathbf{R}^{3}$ and $\mathbf{R}^{4}$. He had previously made similar comments about the odds that random guessing would produce a suitable basis:

Bob: Well, it's comparing two infinities, but like. If it's $\mathbf{R}^{3}$, if they're linearly dependent then they only form like a plane in $\mathbf{R}^{3}$. And there's just one plane out of the infinitely many other planes that are in $\mathbf{R}^{3}$. Basically, it's the odds of the three points [sic; vectors is meant] all being in the same plane in $\mathbf{R}^{3}$, which $\mathbf{R}^{3}$ is just like, it's three-dimensional, and the odds that they're all just in a twodimensional slice of it is much lower. I mean, yeah, I guess you can't come up with odds, because they're infinities, but maybe like $1 \%$ or something. [laughs] Probably less than that.

As discussed earlier, the occurrence of the word "plane" is a key indicator of Geometric thinking; this was the first time it appeared in Bob's interview.

Alan provides another example of Geometric thinking powering intuition. When asked to describe orthogonality, he uses the Abstract mode to provide a "technical definition," then describes his intuition in more Geometric terms with reference to "right angles:"

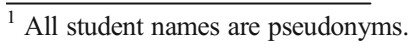


Alan: I usually think about it as being perpendicular, but I think the technical definition is more that the dot product is zero. But I like thinking about it as the directions in $\mathbf{R}^{3}$, just like your $x$ and your $y$ and your $z$ pretty much. And then in $\mathbf{R}^{n}$, you just have $n$ directions... they're sort of at right angles. I don't know how to think about that in $\mathbf{R}^{n}$, but that's what I think about; I just think about $\mathbf{R}^{3}$ and use that as a basis for my thinking about $\mathbf{R}^{n}$.

In contrast to the pilot study, where student thinking was largely balanced between all three modes of thinking, we noted a preponderance of Computational thinking in the present study; indeed, two students provided no evidence of Geometric thinking at all. As a crude indication, although the word "orthogonal" was used multiple times by each student, the specifically Geometric word "perpendicular" was used by only two of the eight students, and the Geometric word "plane" was used by four of the eight. In contrast, five of the eight students in the pilot study provided evidence of Geometric thinking in response to the Michelle question. This surprising (in view of the pilot study) preponderance of Computational thinking is what led us to expand our initial list of research questions to include a more focused question examining students' use of Computational thinking. Our data suggest that students using Computational thinking can generate strategies, formulate justifications, reveal tacit assumptions, and provide several different ways to frame a solution process. We present vignettes of two students who exhibit some of these surprising and productive ways of using Computational thinking. Through these vignettes, we illustrate some of the affordances and pitfalls of Computational thinking, which we summarize at the end of this section.

\section{Bob: Strategy Generation and Computational Justification}

To prompt students to justify their solutions, we included in our protocol the question, "Michelle is skeptical that your solution will always work. How would you convince her?" We had anticipated that this question would prompt Abstract thinking, as this is the language commonly used in theorems and proofs. However, we found that several students were able to produce largely valid justifications using Computational thinking alone. Additionally, several students' Computational thinking inspired the creation of strategies for choosing vectors. The first vignette, featuring a student we call Bob, exemplifies both of these phenomena.

Bob used what we call the Missing Pivots strategy, which he came to formalize as follows: "Form an augmented $4 \times 2$ matrix with $\mathbf{v}$ and $\mathbf{w}$ and row reduce to echelon form. From there, choose two new vectors so that the nonpivotal rows have a nonzero number, and all other numbers in the vector are zero." In particular, Bob used elementary basis vectors to supply the missing pivots.

Recall that, when a matrix is in reduced row echelon form, the leftmost nonzero entry of any row must be 1 , by definition. In the textbook used for this course, and thus in the classroom discourse, these entries are called "pivotal 1s" or "pivots" (some texts call them "leading 1s"). Accordingly, the rows or columns in which the pivotal 1s occur are called pivotal rows or columns. The pivotal columns of the reduced row echelon form $M_{\text {re }}$ of a matrix $M$ correspond to linearly independent columns of $M$. For example, 
if columns 1, 3, and 4 of $M_{\mathrm{re}}$ are pivotal, then columns 1, 3, and 4 of $M$ are linearly independent. In the context of the Michelle problem, the full set of column vectors of $M$ is independent if all columns are pivotal. Bob proposed augmenting the $4 \times 2$ matrix $M_{\mathrm{re}}$ with two additional columns so that each column (and row) in the resulting $4 \times 4$ matrix is pivotal.

Bob's procedure was interesting to us in its own right for its sophistication and novelty; this was not one of the methods we had anticipated students would use. However, Bob's development of this procedure was also noteworthy. He first stated that "if you form a $4 \times 4$ matrix with [the vectors], it will row reduce to the identity in $\mathbf{R}^{4}$. So, you need to choose vectors that are likely to row reduce to pivotal columns." He suggested that "the odds are really good" that pure guess and check will accomplish this, and that including zeroes in the additional vectors will be helpful: "she could include zeroes to make it easier, zeroes being a way to easily show that there's no way you can write it as a combination of the other ones." Specifically, he proposed adding two vectors of the form $\left[\begin{array}{lll}0 & 0 & \# \#\end{array}\right]$ and $\left[\begin{array}{llll}0 & 0 & 0\end{array}\right]$, and successfully checked that this procedure works in a specific numerical example. This solution strategy is reflective of a rich conceptual understanding of the relationship between basis vectors and pivot columns, which Bob accessed here through reasoning Computationally about the row reduction process. Thus, Bob provides us with an example of Computational reasoning as "integration of procedural knowledge with conceptual knowledge" (Baroody et al. 2007, p. 121).

In the course of formalizing this method for Michelle, it emerged that he was tacitly assuming that the first two columns $\mathbf{v}$ and $\mathbf{w}$ of the matrix reduce to the standard basis vectors $\mathbf{e}_{1}$ and $\mathbf{e}_{2}$. When the interviewer questioned this assumption, suggesting a counterexample $\mathbf{v}=\mathbf{e}_{1}$ and $\mathbf{w}=\mathbf{e}_{3}$, Bob realized that he needed to use the first two columns to ascertain which pivots are missing and must be supplied by the two additional vectors. Thus, the Missing Pivots strategy emerged as a refinement of simple guess and check methods motivated by concrete counterexamples to an insufficiently general tacit assumption. His construction of the procedure could be described as reverse-engineering the check: by using Computational reasoning to think about the test his vectors must pass, he was able to engineer vectors that are guaranteed to pass it. Therefore, an affordance of Computational reasoning is the possibility of testing and refining methods and assumptions against well-chosen examples and counterexamples.

Next, the interviewers asked him a question intended to elicit justification: "Michelle is skeptical that your method will work; how would you convince her?" Bob's justification was entirely Computational:

Bob: Well, by the definition of linear independence, their matrix has to row-reduce to the identity. All the columns will be pivotal. So, by using these facts about linear independence and pivotal columns, this procedure is a way to find two more columns that will be pivotal columns independent of the other ones already found. With the two vectors she already has, she has two pivotal columns here, and they both represent pivotal ones. It's just a way to find the other two columns that won't 
form the same pivotal row as another one, so that they'll all be independent.

We note the presence of strongly Computational language such as "row-reduce" and "procedure." Additionally, we argue that the notion of pivots on which this argument relies is so closely linked to the row reduction algorithm that it is a de facto sign of Computational thinking.

This technique of justification by analysis of an algorithm is a particularly valuable way of constructing formal justifications. Many proofs in linear algebra proceed in a similar fashion; for instance, the usual proof that more than $n$ vectors in $\mathbf{R}^{n}$ cannot be linearly independent proceeds by forming a matrix whose columns are these vectors, row reducing, and arguing that the shape of the resulting echelon form implies that one vector is a linear combination of the others. The fact that students are capable of producing such justifications, as evidenced by Bob and others in our study, is perhaps an argument for teachers to highlight this method when discussing proof techniques.

It is worth noting that Bob's justification falls slightly short of being a fully correct proof. In particular, say that the completed basis consists of the given vectors $\mathbf{v}$ and $\mathbf{w}$ and the additional vectors $\mathbf{x}$ and $\mathbf{y}$. The vectors supplementing the row-reduced forms of $\mathbf{v}$ and $\mathbf{w}$ are technically the row-reduced forms of $\mathbf{x}$ and $\mathbf{y}$, and should have the inverse row operations applied to them to yield the original $\mathbf{x}$ and $\mathbf{y}$. In Bob's case, where the additional vectors are elementary basis vectors, the particular inverse row operations that would be applied would leave the additional vectors unchanged, but to be called a fully correct proof, his argument should have addressed this complication. Our data do not provide clear evidence of whether he was aware of this issue. In any event, the Computational justification Bob produced was correct as far as it went, and was most of the way to a complete proof.

\section{Greg: Numerical Examples and Framing}

The work of a student called Greg illustrates several more facets of students' productive Computational thinking. Here we first discuss Greg's solution procedure with minimal editorial comment, then offer two possible and related explanations of his actions.

To orient the reader, we briefly discuss the mathematics underlying what we called the Unknown Columns strategy, illustrated by Greg in Fig. 1. This strategy proceeds as follows: augment $\mathbf{v}$ and $\mathbf{w}$ together with vectors composed entirely of variables, and row reduce until the first two columns are $\mathbf{e}_{1}$ and $\mathbf{e}_{2}$. At this point, the third and fourth columns are partially row reduced. To continue the row reduction process from here, one would need to divide the third column by the diagonal entry; thus, for $\mathbf{x}$ to be linearly independent of $\mathbf{v}$ and $\mathbf{w}$, it suffices that the diagonal entry be nonzero. This puts a constraint on the components of the original vector $\mathbf{x}$; a similar constraint on the values of the original vector $\mathbf{y}$ emerges from continuing the row reduction process further.

As stated above, Greg used this Unknown Columns strategy: he augmented $\mathbf{v}$ and $\mathbf{w}$ with vectors $\mathbf{x}$ and $\mathbf{y}$ composed entirely of variables (see Fig. 1), and performed row 


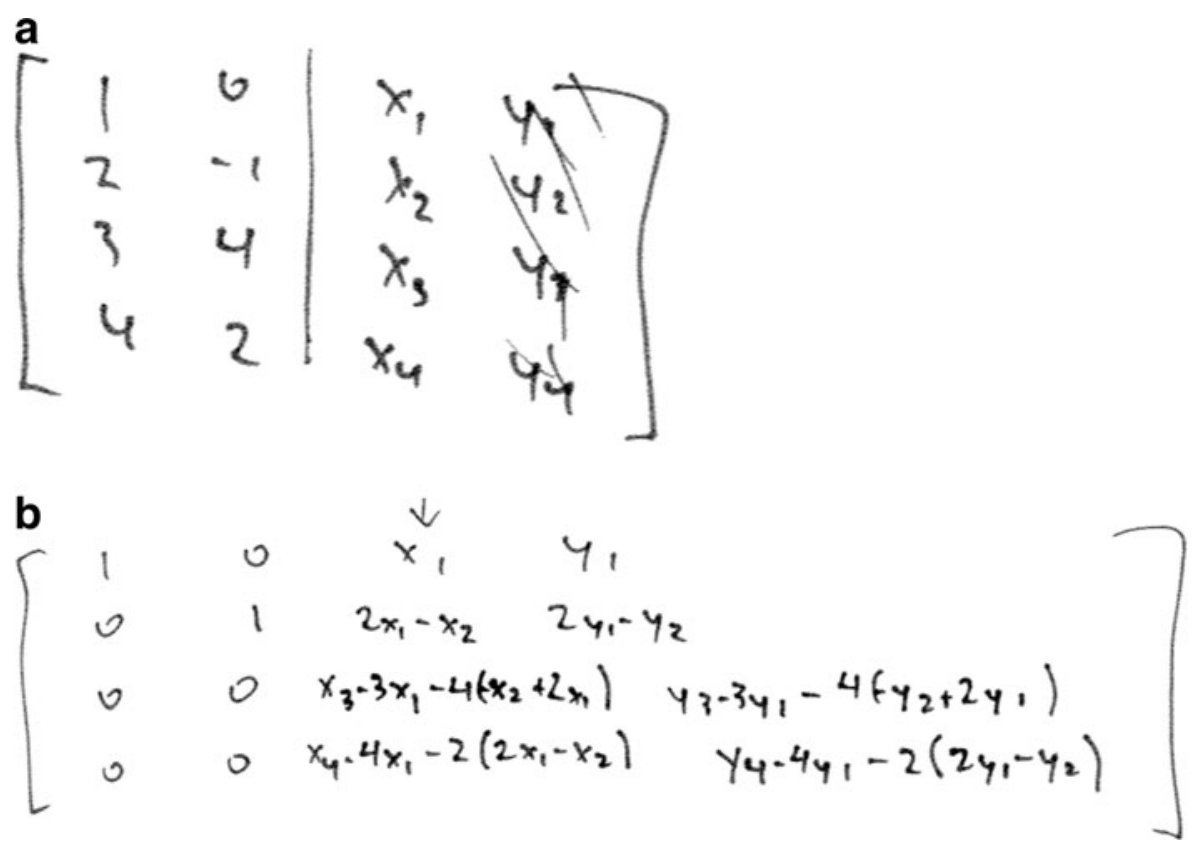

Fig. 1 Greg's unknown columns matrix, initial and row-reduced

operations on the resulting $4 \times 4$ matrix until the first two columns were reduced to $\mathbf{e}_{1}$ and $\mathbf{e}_{2}$. He obtained the following matrix:

He then said, "These two [the diagonal entries] should be 1, and those [the offdiagonal entries] should be zeroes... because for these columns to be linearly independent, it would row-reduce to the identity matrix." This reasoning gave him the following four equations for the third column $\mathbf{x}$, and similar equations for the fourth column $\mathbf{y}$ :

$$
\begin{aligned}
& x_{1}=0 \\
& 2 x_{1}-x_{2}=0 \\
& x_{3}-11 x_{1}+4 x_{2}=1 \\
& x_{4}-8 x_{1}+2 x_{2}=0
\end{aligned}
$$

(These equations are overly restrictive, in that they are sufficient but not necessary for the third column to be linearly independent of the first two. In particular, the only necessary condition is that $x_{3}-11 x_{1}+4 x_{2}$ is nonzero.)

The interviewers were interested to see how far he could push this line of reasoning, and asked him, "Can you give numerical vectors that satisfy these equations that you've got?" He obliged, and found that this system of equations uniquely determines the

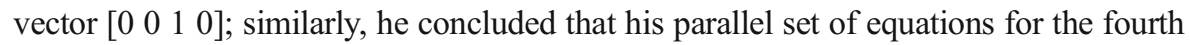
column $\mathbf{y}$ uniquely determines the vector $\left[\begin{array}{llll}0 & 0 & 0 & 1\end{array}\right]$. (This is the precise sense in which the equations Greg wrote are overly restrictive; they uniquely determine $\mathbf{e}_{3}$ and $\mathbf{e}_{4}$, which are valid solutions to this problem, but not the only ones.) This appeared to violate his expectations, inasmuch as he asked, "Is there a way to take this back to the non-row-reduced form?" He thus seemed to believe that $\left[\begin{array}{llll}0 & 0 & 1 & 0\end{array}\right]$ was the row- 
reduced version of the original $\mathbf{x}$ vector, and was unsure how to recover the original vector. He also said he was sure that this row-reduced version of $\mathbf{x}$, denoted $\mathbf{x}_{\mathrm{r}}$, is independent of $\mathbf{v}_{\mathrm{r}}$ and $\mathbf{w}_{\mathrm{r}}$, but perhaps not independent of $\mathbf{v}$ and $\mathbf{w}$ themselves; further, he was sure that $\mathbf{x}$ (the original, "non-row-reduced" version) would be independent of $\mathbf{v}$ and $\mathbf{w}$.

Later in the interview, the interviewers asked Greg to reflect on how he would validate a proposed third vector, and gave him a numerical example, [1 $\left.\begin{array}{lll}-1 & 0 & 0\end{array}\right]$. He augmented $\mathbf{v}$ and $\mathbf{w}$ with this vector and proceeded to row-reduce until his first two columns were $\mathbf{e}_{1}$ and $\mathbf{e}_{2}$. At this point, his third row was $[0$ $0 \mid-15]$ (see Fig. 2 below). He seemed unsure how to interpret this result, saying, "I'm actually kinda confused about what this tells me. Um... Did I make a mistake?"

Once the interviewers assured him that he had not made a computational error, he reasoned further:

Greg: Well, actually, if I continue row-reducing this, then I would get a 1 here [in place of the -15 in the final column], and then that would make it unsolvable. ... And then, that would mean that this can't be a linear combination of these two. So then, it's not in the span.

Int: And is that good or bad, for purposes of this problem?

Greg: That's good. So then, this could be an additional vector for a basis.

Int: And just what about your answer says that?

Greg: If I continue row-reducing this, which is something that I also blanked out on, then I would get a 1 in the final column, and that states that 0 times whatever plus 0 times whatever, so on, equals 1 , which is not true. So searching for solutions is what this is, so therefore there are no solutions.

Next, the interviewers asked Greg to reflect on what this example would mean for his original solution process (i.e., his Unknown Columns method). He realized that he had imposed too many constraints on the variables:

Greg: I should have known that if this thing that ended up in the $\mathrm{x}_{3}$ spot [i.e., the diagonal entry], if this was equal to 1 , then there'd be no solution. So this is what I want to satisfy. ... Really, that's the only important thing, basically. This stuff

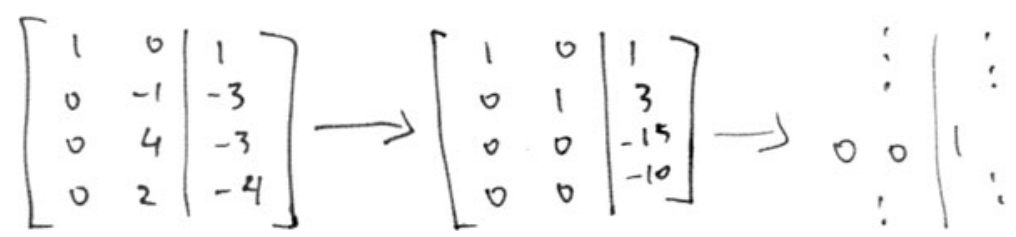

Fig. 2 Greg's work on validating a proposed third vector 
[i.e., the constraints on the non-diagonal entries] is not important. It's just this [constraint on the diagonal entry].

Int: What do you mean it's just that that's important?

Greg: This is the only thing that would determine whether or not my new column would be in the span. ... And actually it's not just "equal to 1," it's "not equal to zero." So it can be any number.

Greg then replaced his previous conditions with the new condition, depicted in Fig. 3.

It is visible in this Figure that Greg first wrote " $=1$ " but then replaced this with " $\neq 0$ " (thus replacing his original overly-restrictive condition with the correct necessary condition discussed earlier). He then chose numbers for the $x_{\mathrm{i}}$ 's that satisfied the given constraint, and seemed much more satisfied with this solution than with his previous solution.

Now that we have recounted Greg's story, we propose two related explanations for his "a-ha moment" and subsequent correction of his strategy. First, the numerical example may have shown Greg that he was not done with his row reduction process. When row-reducing his matrix, he stopped as soon as the first two columns were in the form he wanted, and seemed to believe that the matrix was then in reduced row echelon form and should thus be equal to the identity matrix; note, for instance, that he obtains

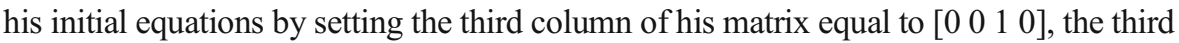
column of the identity matrix. He did not seem to realize that there was more work he needed to do to reach this point (i.e., dividing the third row by the diagonal entry to create a pivotal 1, etc.); this work may have been obscured by the algebraic complexity of his approach. In the numerical example, he obtained [ $\left.\begin{array}{llll}1 & 3 & -15 & 10\end{array}\right]$ as his third column at the same point in the row reduction process, which seems to have helped him realize that the third column of his Unknown Columns matrix need not be equal to $\left[\begin{array}{llll}0 & 0 & 1 & 0\end{array}\right]$, allowing him to relax the constraints on the variables accordingly. It thus appears that numerical examples can illuminate what purely algebraic manipulations can obscure; however, both of these are examples of Computational thinking, since they both involve reasoning about the row reduction process. It is thus evident that Computational thinking can take many forms, and that one form can be used to help make sense of another. It is also interesting that both Greg's error (not realizing that he was not done with the row reduction process) and its resolution (by way of examining a specific numerical example) came from his use of Computational reasoning.

Additionally, the algebraic complexity of Greg's work may have hindered his thinking in another way. As shown by his desire to find the "non-row-reduced form" of his initial solution, he seemed to have lost track of the meaning of the variables he introduced; by definition, they are the entries in the original matrix, not its reduced form, and he had already undone the row reduction process by solving the equations he

$$
x_{3}-3 x_{1}+4 x_{2}-8 x_{1} \neq 0
$$

Fig. 3 Greg's constraint 
had accumulated in the third and fourth columns. Again, it appears that the numerical example helped him realize what his variables had originally meant.

Second, once Greg realized that he was not done, his thinking shifted from solving a system of equations (induced by setting his third column equal to [ $\left[\begin{array}{llll}0 & 0 & 1 & 0\end{array}\right]$ ) to finding and satisfying constraints (induced by saying that the diagonal entry should be nonzero). This is an example of a shift in what we call a framing; that is, a student's implicit mental expectation for how a computation should go. Greg shifted from thinking of equations, where the expected action is to solve and the expected outcome is a specific solution, to thinking of constraints, in this case inequalities, where the expected action is to satisfy and the expected outcome is a range of possible solutions. Both framings are useful at times, but for Greg, the framing as equation was less appropriate for the situation and seemed to cause him difficulty. The framing as equation led him to the (apparently unsatisfactory) conclusion that adding $\mathbf{e}_{3}$ and $\mathbf{e}_{4}$ should be the only solution to the problem; while this is a correct solution, it is not the most general form. By imposing unnecessarily strong constraints, he obtained an overly restricted answer; by shifting his framing and relaxing the constraint, he obtained a much more general form.

Our use of the construct framing is broadly consistent with its prior use in education research and other fields of social science. Hammer et al. (2005) define a frame (p. 98) as "a set of expectations an individual has about the situation in which she finds herself that affect what she notices and how she thinks to act." In addition to frames for social situations, these authors mention epistemological frames that describe the methods an individual expects to use to construct or justify knowledge. We suggest that students have expectations for the sequence of steps a computation will follow and the outcomes of at least some of these steps. This notion is linked to those of scripts, schemas, monitoring, and metacognition (see, e.g., Polya 1945; Davis 1984; Schoenfeld 1992; Asiala et al. 1996). The frame may include actions to be taken if the expectations are not met, but a sufficient deviation from expectations may trigger a shift of framing instead.

The Unknown Columns solution method was attempted by three other students, but not completed due to its algebraic complexity. One of these three students, Alan, exhibited behavior that we interpret as another shift in framing. In the course of row-reducing the matrix, he freely divided rows by algebraic expressions that appeared in their pivotal locations, so as to obtain pivotal 1s there. He was not initially aware of the fact that this amounted to assuming the pivotal entries to be nonzero and thus the matrix to be invertible. When he arrived at the identity matrix as the row-reduced form, he was surprised. His framing, or expectation, perhaps based on experience with similar problems when a basis for a subspace was to be found, was that there would be free variables remaining at the end of the reduction, which could be chosen so as to obtain the desired basis. That is, he expected the constraints on the possible additional vectors to appear at the end of the computation, in terms of the free variables, rather than along the way, in the requirements that certain entries be nonzero. His surprise caused him to reorient his framing and focus his attention on the expressions he had divided by. He was experimenting with setting them equal to specific nonzero values when the interviewer moved on due to time constraints. 


\section{Summary of Affordances and Pitfalls of Computational Thinking}

We now move on from the close examination of these two vignettes to present some more general results about how our students used Computational thinking. Rather than comprehensively examine each student's uses of Computational thinking, we here present as a more general summary a list of the affordances and pitfalls of Computational thinking we identified in our students' thinking, and provide brief examples of each. We noted the following affordances of Computational thinking:

1. Working out an example to provide a general orientation to an unfamiliar problem: before being presented with specific vectors for $\mathbf{v}$ and $\mathbf{w}$, Alan invented his own numerical examples to see how the other two vectors might be related.

2. Searching for a known algorithm that applies to the situation, or evaluating the applicability of a known algorithm: several students thought about using the GramSchmidt process, but eventually discarded this idea when they realized it required a different set of inputs than the situation afforded.

3. Recognizing when systems of equations have no solution, a unique solution, or infinitely many solutions: Greg reasoned that his $\left[\begin{array}{ll}0 & 0\end{array} \mid 15\right]$ row indicates that there is no solution, so the vector is not in the span of $\mathbf{v}$ and $\mathbf{w}$; another student, Hal, said that there are infinitely many bases that satisfy Michelle's requirements because there is not just one solution to a certain equation.

4. Clarifying a more general approach with a numerical example: Greg was confused by his Unknown Columns method until he worked through the validation of [ $\begin{array}{lll}1 & -1 & 0\end{array}$ $0]$ as a proposed third vector.

5. Making choices to simplify computations or reasoning: Bob proposed the strategic positioning of zeroes to "make it easier" to show that the guessed vectors are independent of the original vectors.

Naturally, Computational thinking was not a panacea; some students encountered difficulties that emerged directly from their use of Computational thinking. Here are some of the pitfalls of this way of thinking that we observed:

1. Thinking that coefficients in linear combinations must be integers: when checking whether or not a third vector was linearly independent of two others, Alan only checked integer combinations of the first two until challenged by the interviewer. Students' default concept image of a "number" is often a (positive) integer, perhaps based on experience with oversimplified textbook examples (the "natural number bias;" see, e.g., Christou and Vosniadou 2012).

2. Dividing by variable expressions without imposing the constraint that they must be nonzero: during the row reduction process in the Unknown Columns solution method, Alan divided a row by the expression $\left(x_{3}-3 x_{1}\right)+4\left(x_{2}-2 x_{1}\right)$ which appeared in the diagonal entry. He then continued row reducing until he obtained the identity matrix, and then became confused that he had no constraints on his variables, neglecting the fact that dividing by variable expressions would have introduced constraints earlier.

3. Becoming overwhelmed by sheer algebraic complexity, e.g., many variables: Carl attempted the Unknown Columns method (which introduces eight variables 
representing the entries of these columns) but did not persist to a solution he regarded as satisfactory because dividing by the diagonal entry would lead to overly complicated entries elsewhere.

4. Circular substitutions leading to vacuous statements: when attempting the orthogonality approach described below, Carl made circular substitutions that led him to the equation $0=0$.

5. Narrow focus on algorithmic procedures: several students thought that GramSchmidt was the only way to produce orthogonal vectors.

6. Uncertainty about meaning of variables, leading to difficulty in interpreting results: as described above, Greg lost track of the meaning of his variables, leading him to be confused about whether or not his result was row-reduced.

7. Failing to realize when an algorithm has (not) been run to its proper completion: Hal stopped row reducing before obtaining the identity matrix, leading him to mistakenly conclude that the two vectors he chose formed a linearly dependent set with the first two.

8. Narrowly framing a computation in terms of solving an equation, leading to an expectation that the solution will be unique. We interpreted Greg's thinking as a shift away from this narrow framing. Other sorts of algorithms may be subject to this pitfall as well; for example, the standard algorithm for computing a basis for the null space (say) of a matrix may foster the belief that this is the only basis for that space.

Many of these dangers will likely be familiar to any reader acquainted with students' thinking in linear algebra.

\section{Responses to the Suggested Orthogonality Approach}

Contrary to our expectations based on the pilot study, no student spontaneously suggested finding vectors orthogonal to both $\mathbf{v}$ and $\mathbf{w}$. When the interviewer proposed this approach (as having been mentioned by "another student") most students agreed that it would work and translated it into some set of equations but generally could not carry it through to a complete solution. They also did not shift to Geometric thinking for guidance, but remained in the Computational or Abstract mode, and this likely contributed to their difficulties.

One student wrote the equations stating that the new vectors should have zero dot products with the original pair, e.g., $\mathbf{v} \mathbf{x}=0$, but tried to manipulate them in that Abstract form as opposed to Computationally introducing the components of the vectors as variables. Two were concerned that the initial vectors $\mathbf{v}$ and $\mathbf{w}$ were not orthogonal to each other, perhaps thinking that orthogonality is a transitive property. Geometric thinking would clarify that the new vectors need to be orthogonal to the plane of $\mathbf{v}$ and $\mathbf{w}$, the particular basis in this plane being irrelevant. An indicator of the absence of Geometric thinking is that all students used the term orthogonal rather than perpendicular when reasoning about this proposed solution process. Students who formulated the equations in components could make further progress, sometimes obtaining a partial solution, but did not obtain a general solution or clearly understand how many solutions there would be. Some did not write the complete set of orthogonality requirements, and one unnecessarily required the two new vectors to be orthogonal 
to each other (making his system of equations nonlinear). In this approach to the problem, purely Computational thinking in the absence of coordination with the Geometric mode was insufficient for most students, consistent with our original hypothesis.

\section{Discussion}

Some authors, and some instructors, may view Computational thinking as mathematically unsophisticated, a form of procedural knowledge in the narrow sense (Hiebert and Lefevre 1986). Our data show that this is not necessarily the case; the students in our study were able to use Computational thinking in a variety of sophisticated, productive, and reflective ways, including generating Computational justifications for claims and making strategic choices to limit the complexity of their calculations. Our work thus joins the body of literature presenting an optimistic picture of student reasoning in linear algebra (e.g., Possani et al. 2010; Wawro 2014; Wawro et al. 2012), and provides evidence for the utility of Computational reasoning. We also hope to have provided evidence for the analytic usefulness of including reasoning processes within the construct of Computational thinking.

The fact that students in our study largely remained in the Computational mode of thinking throughout the interviews could be viewed as confirmation of Hillel's and Sierpinska's observations that they have difficulty switching flexibly between modes even when this would be helpful. However, the question remains, why did these students use Computational thinking so much more than those in our pilot study (Wawro et al. 2011)? Both groups of students took the same course, with the same textbook, and were interviewed at the end of the class. While our data provide no definitive answers, we can offer some conjectures. First, the class in the present study was taught with a heavy emphasis on computations. Many of the central problems of linear algebra were framed in terms of finding solutions to systems of equations; this is also the approach taken by the textbook (Hubbard and Hubbard 2009). In contrast, the students in the pilot study were taught by a different instructor who takes a more Geometric approach to the subject.

Second, the structure of the interview may have privileged Computational ways of thinking. To minimize feelings of frustration and to smooth the way for students to produce as much interesting mathematics as possible, we gave students the concrete numerical vectors fairly early on in the interview, which may have led students to more Computational thinking than their natural inclination. We could well have waited until later to provide the concrete vectors, and pushed harder for non-numeric solutions to the problem.

It is also possible that our students have not yet developed the ability to use Geometric thinking in higher-dimensional contexts. Framing the Michelle problem in $\mathbf{R}^{4}$ may have precluded this mode of thinking. We could have explored this possibility by asking them to re-situate the problem in $\mathbf{R}^{3}$.

We had hoped to investigate students' use of multiple modes of thinking, and the nature of the coordination or interaction between them. What are the "boundaries" between the modes and how should thinking near these boundaries be characterized? What can prompt students to switch modes, and how do insights obtained in one mode 
affect thinking in another? Do difficulties in coordinating representations between different modes outweigh the benefits obtained from flexibility? These questions cannot be answered with the data we have reported in this paper, and thus remain for future work. The interview protocol may need to be redesigned to provide more opportunities for students to switch modes of thinking, or include questions that actually prompt such a switch.

We may recommend a few pedagogical implications of our work. First, the success of students in constructing Computational justifications by analysis of algorithm (and the frequent presentation of such proofs in linear algebra textbooks) may imply that instructors should foreground this technique in classes with a proof component. Textbooks normally justify each new algorithm with a proof that it does compute what it claims to compute, and the structure of such proofs could be emphasized. Other uses of the technique, such as the proof by row-reduction that a linearly independent set in $\mathbf{R}^{\mathrm{n}}$ contains at most $n$ vectors, could be highlighted. Second, our results suggest that instructors may wish to adopt a more positive orientation toward computational and procedural thinking in general, notice when their students are using it productively, and help them grow more expert-like in the ways they use it. In particular, instructors may be more explicit about the notions of framing and metacognition. Students could be encouraged to anticipate the output of an algorithm they employ, monitor its steps as it progresses to check consistency with expectations, and consider an Abstract or Geometric viewpoint for making sense of unexpected results.

Finally, our data imply that Computational reasoning in linear algebra is an opportunity for students to develop and utilize deep procedural knowledge (Baroody et al. 2007). While not all Computational reasoning is particularly deep, and while our sample is composed of students in a high-achieving population and is therefore not representative of the general population of linear algebra students, our results are an existence proof. Our students' use of procedural elements such as framing and reverseengineering in the service of activities such as strategy generation, justification, and troubleshooting is evidence that linear algebra students can, perhaps with appropriate scaffolding from their instructors, use Computational reasoning in ways that blend procedural and conceptual knowledge. Our data contain many examples of what the sophisticated blending of procedural and conceptual knowledge might look like. We hope that other researchers and practitioners will join us in recognizing and researching productive ways students might develop and use deep procedural knowledge.

\section{Appendix 1: Interview Protocol}

The task is posed in the relatively abstract form: Michelle would like to create a basis for $\mathbf{R}^{4}$. She has already listed two vectors $\mathbf{v}$ and $\mathbf{w}$ that she would like to include in her basis, and wants to add more vectors to her list until she obtains a basis. What instructions would you give her on how to accomplish this? Students are provided with this task in written form, and scratch paper to use.

After students have suggested a solution method, or if they are having difficulty producing one: Suppose the specific vectors $\mathbf{v}$ and $\mathbf{w}$ Michelle has chosen are $\left[\begin{array}{lll}1 & 2 & 3\end{array}\right]$ and $\left[\begin{array}{lll}0 & -1 & 4\end{array}\right]$. Can you show how your procedure would work with these vectors? Would you like to revise your procedure after trying it with these vectors? 
Questions to elicit formalization and justification: Michelle is only available via email; how would you write down your procedure to communicate it to her? Suppose Michelle would like to find another basis containing $\mathbf{v}$ and $\mathbf{w}$; can she use your method to find another? Michelle is skeptical that your method will work; how would you convince her?

Questions for students who propose guess-and-check methods: Suppose you are a very unlucky guesser; is there a strategy for making better guesses? How does row reduction tell you that the vectors form a basis? Suppose Michelle wants to guess additional vectors one at a time; can she check the third vector before guessing the next?

Suggesting an alternate method, potentially eliciting geometric thinking: Another student suggested that since orthogonal vectors are independent, Michelle should pick vectors orthogonal to those she already has. What do you think of this idea?

Additional questions: How many bases are there that meet Michelle's requirements? Can you describe them? How many dimensions of freedom are there in choosing such bases? Can Michelle begin with any two vectors $\mathbf{v}$ and $\mathbf{w}$, or does she need to be careful in choosing them?

Within the above framework, the interviewer was free to ask additional questions exploring students' thinking about the methods they employed. Although it was not his purpose to help the students solve the problem, some of the questions caused students to reflect on difficulties they had encountered and were therefore of assistance.

\section{Appendix 2: Solution Methods for the Michelle Problem}

When we designed our study, we listed possible solution methods that we expected students might use. These are named in boldface below, and are followed by the additional unanticipated methods our students used. The numbers of students observed to employ each method is given. Note that some students used multiple approaches.

1. Guess and Check (5 students). Simply guess two vectors $\mathbf{x}$ and $\mathbf{y}$ to supplement $\mathbf{v}$ and $\mathbf{w}$ in forming a basis. Check that the set of four do form a basis, for example by row reducing a $4 \times 4$ matrix having these vectors as columns. This method succeeds with probability one, that is, unless the guesses are very unlucky.

2. Abstract Proof (0 students). Since the span of $\mathbf{v}$ and $\mathbf{w}$ is two-dimensional, choose any vector $\mathbf{x}$ not in this span. Then choose any vector $\mathbf{y}$ not in the threedimensional span of $\mathbf{v}, \mathbf{w}, \mathbf{x}$. This is a proof of existence of the desired basis, in the Abstract language. To translate it into a practical algorithm, Computational thinking is needed to specify a method for making the required choices. For example, the span of $\mathbf{v}$ and $\mathbf{w}$ can be characterized by row reducing a $4 \times 2$ matrix having those columns, to find a simple basis for this span. Then find by inspection a vector that is not a linear combination of these basis vectors. Or, augment this matrix with a third column of unknowns and row reduce to determine the conditions on the unknowns for this third column not to be a linear combination of the first two. Several students stated the Abstract definition of a basis, but did not appear to extend this into a proof by, for example, arguing that there are vectors in $\mathbf{R}^{4}$ outside the two-dimensional span of $\mathbf{v}$ and $\mathbf{w}$. 
3. Orthogonality ( 0 students). Find all vectors orthogonal to both $\mathbf{v}$ and $\mathbf{w}$ (Geometric thinking), for example by computing the kernel of the $2 \times 4$ matrix having those rows. Choose two independent vectors in this kernel to complete the desired basis. This idea was suggested to students during the interview, but none used it spontaneously. This was in contrast to the pilot study, in which many students proposed it as their initial response.

4. Modified Linear Combination (1 student). Take some linear combination of $\mathbf{v}$ and $\mathbf{w}$, and alter one component of the resulting vector. This gives a vector $\mathbf{x}$ that is (almost certainly) independent of $\mathbf{v}$ and $\mathbf{w}$. Repeat with some linear combination of $\mathbf{v}, \mathbf{w}$, and $\mathbf{x}$.

5. Unknown Columns (4 students). Create a $4 \times 4$ matrix whose columns are $\mathbf{v}, \mathbf{w}$, and two columns of unknowns representing the desired additional vectors $\mathbf{x}$ and $\mathbf{y}$. Row reduce to determine the conditions on the unknowns that make the four columns independent. Choose values satisfying these conditions.

6. Missing Pivots ( 2 students). Row reduce the $4 \times 2$ matrix having columns $\mathbf{v}$ and $\mathbf{w}$, noting which rows of the reduced matrix contain pivots. Then supply two additional columns having pivots in the complementary rows (for example, two of the standard basis vectors would accomplish this). The reduced columns are then independent, and by reversing the sequence of steps in the row reduction one obtains four independent columns $\mathbf{v}, \mathbf{w}, \mathbf{x}, \mathbf{y}$ forming a basis. The choice of standard basis vectors is particularly convenient because they will often not change under the reduction steps.

\section{References}

Asiala, M., Brown, A., DeVries, D., Dubinsky, E., Mathews, D., \& Thomas, K. (1996). A framework for research and curriculum development in undergraduate mathematics education. In A. Schoenfeld, J. Kaput, \& E. Dubinsky (Eds.), Research in collegiate mathematics education II, CBMS issues in mathematics education (pp. 1-32). Providence, RI: American Mathematical Society.

Baroody, A. J., Feil, Y., \& Johnson, A. R. (2007). An alternative reconceptualization of procedural and conceptual knowledge. Journal for Research in Mathematics Education, 38(2), 115-131.

Carlson, D. (1993). Teaching linear algebra: Must the fog always roll in? College Mathematics Journal, 24, 29-40.

Celik, D. (2015). Investigating students' modes of thinking in linear algebra: the case of linear independence. International Journal for Mathematics Teaching and Learning. Retrieved from http://www.cimt. plymouth.ac.uk/journal/.

Christou, K., \& Vosniadou, S. (2012). What kinds of numbers do students assign to literal symbols? aspects of the transition from arithmetic to algebra. Mathematical Thinking and Learning, 14(1), 1-27.

Davis, R. B. (1984). Learning mathematics: The cognitive science approach to mathematics education. Norwood, NJ: Ablex.

Dogan-Dunlap, H. (2010). Linear algebra students' modes of reasoning: geometric representations. Linear Algebra and its Applications, 432, 2141-2159.

Dorier, J.-L., \& Sierpinska, A. (2001). Research into the teaching and learning of linear algebra. In D. Holton (Ed.), The teaching and learning of mathematics at university level: An ICMI study (pp. 255-273). Dordrecht, Netherlands: Kluwer.

Ginsburg, H. P. (1997). Entering the child's mind: The clinical interview in psychological research and practice. New York, NY: Cambridge University Press. 
Hammer, D., Elby, A., Scherr, R. E., \& Redish, E. F. (2005). Resources, framing, and transfer. In J. Mestre (Ed.), Transfer of Learning from a modern multidisciplinary perspective (pp. 89-119). Greenwich, CT: Information Age Publishing.

Hiebert, J., \& Lefevre, P. (1986). Conceptual and procedural knowledge in mathematics: An introductory analysis. In J. Hiebert (Ed.), Conceptual and procedural knowledge: The case of mathematics (pp. 1-27). Hillsdale, MI: Erlbaum.

Hillel, J. (2000). Modes of description and the problem of representation in linear algebra. In J.-L. Dorier (Ed.), On the teaching of linear algebra (pp. 191-207). Dordrecht, Netherlands: Kluwer.

Hubbard, J., \& Hubbard, B. B. (2009). Vector calculus, linear algebra, and differential forms: A unified approach. Ithaca, NY: Matrix Editions.

Maracci, M. (2008). Combining different theoretical perspectives for analyzing students' difficulties in vector spaces theory. ZDM Mathematics Education, 40, 265-276.

Polya, G. (1945). How to solve it. Princeton, NJ: Princeton University Press.

Possani, E., Trigueros, M., Preciado, J. G., \& Lozano, M. D. (2010). Use of models in the teaching of linear algebra. Linear Algebra and its Applications, 432, 2125-2140.

Schoenfeld, A. H. (1992). Learning to think mathematically: Problem solving, metacognition, and sense making in mathematics. In D. Grouws (Ed.), Handbook of research on mathematics teaching and learning (pp. 334-370). New York, NY: Macmillan.

Sierpinska, A. (2000). On some aspects of students' thinking in linear algebra. In J.-L. Dorier (Ed.), On the teaching of linear algebra (pp. 209-246). Dordrecht, Netherlands: Kluwer.

Star, J. R. (2005). Reconceptualizing procedural knowledge. Journal for Research in Mathematics Education, 36(5), 404-411.

Stewart, S., \& Thomas, M. O. J. (2010). Student learning of basis, span and linear independence in linear algebra. International Journal of Mathematical Education in Science and Technology, 41(2), 173-188.

Strauss, A., \& Corbin, J. (1994). Grounded theory methodology: An overview. In N. K. Denzin \& Y. S. Lincoln (Eds.), Handbook of qualitative research (pp. 273-285). Thousand Oaks, CA: Sage Publications.

Wawro, M. (2014). Student reasoning about the invertible matrix theorem in linear algebra. ZDM Mathematics Education, 46, 389-406.

Wawro, M., Sweeney, G. F., \& Rabin, J. M. (2011). Subspace in linear algebra: investigating students' concept images and interactions with the formal definition. Educational Studies in Mathematics, 78(1), 1-19.

Wawro, M., Rasmussen, C., Zandieh, M., Sweeney, G. F., \& Larson, C. (2012). An inquiry-oriented approach to span and linear independence: the case of the magic carpet ride sequence. PRIMUS, 22(8), 577-599. 